\title{
Managing the acute painful episode in sickle cell disease
}

\author{
B. Kaya \\ Consultant Haematologist, Royal London Hospital, UK
}

\begin{abstract}
Sickle cell pain is a complex but frequently experienced symptom. Acute painful events in children can be managed effectively in the community with appropriate support and education. If hospital management is required, rapid access to a consistent, reliable and safe analgesic pathway is recommended to ensure a good standard of care. Use of oral opiates in addition to short acting easily administrable forms of analgesia and strict adherence to protocoled monitoring will enable the acute event to be well managed and the negative pain experience minimised. An important part of the outpatient evaluation is determining the impact pain events are having on the child's quality of life. Addressing psycho-social aspects, defining and modifying precipitating factors, if any are identified, and having a holistic approach to pain management is helpful. Education and use of self-management techniques can also be productive. Use of sickle modifying interventions such as hydroxycarbamide therapy or transfusion therapy can have a significant impact on reducing the severity and frequency of the acute pain event.
\end{abstract}

\section{Introduction}

The acute painful event is the commonest manifestation of sickle cell disease. Although this frequent complication is mostly experienced and managed at home, it remains one of the primary reasons for patients seeking medical intervention in the hospital environment.

For many patients however, attending hospital for management of this complication, the experience is suboptimal with many patients

Correspondence: B. Kaya, Consultant Haematologist, Royal London Hospital, UK.

Key words: sickle disease, pain.

CCopyright B. Kaya, 2011

Licensee PAGEPress, Italy

Thalassemia Reports; 1(s2):e26

doi:10.4081/thal.2011.s2.e26

This article is distributed under the terms of the Creative Commons Attribution Noncommercial License (by-nc 3.0) which permits any noncommercial use, distribution, and reproduction in any medium, provided the original author(s) and source are credited.

Parts of this work were presented at the "12th International Conference on Thalassemia and Hemoglobinopathies", Antalya (Turkey), 11-14 May 2011. reporting delay in assessment, initiation of therapy and repeated dosage. Often monitoring is inadequate and support limited. This negative experience of pain management can increase anxiety, prolong the pain episode and admission and subsequently jeopardise the relationship between the patient, family or carer, and health care professional. Suboptimal treatment is also associated with significant morbidity and mortality.

This was recently highlighted in a UK national confidential report into patient outcomes and deaths, the NCEPOD report. This report reviewed the circumstances around deaths in patients with haemoglobinopathies and was interested in determining remediable factors in the prophylaxis and management of the clinical syndromes caused by sickle cell disease as well as thalassaemia. A number of cases of preventable death were attributed to mismanagement of a painful episode.

The report highlighted the importance of clear local protocols and pathways for managing pain with a particular emphasis on monitoring following administration of opiate analgesia.

This review will illustrate one pain management protocol that has been successfully employed in the management of children presenting with a painful event in East London, UK.

\section{Background to the acute pain event}

Sickle cell pain is likely multi-factorial in aetiology with vaso-occlusion playing a part in the pathogenesis but other less well-characterised factors also contributing to this process.

Children with acute sickle cell pain can present with pain of variable intensity and severity with differing locations and an understanding of the clinical presentation can be instrumental in the management. In young children dactylitis may be evident and acute pain can also be a manifestation of a more complicated process such as osteomyelitis.

Appropriate assessment of pain using validated age appropriate pain scoring methods can be helpful in the initial evaluation as well for determining response to therapy and an awareness of the emerging evidence of sickle cell pain having distinct phases is also important when devising pain management strategies.

\section{Management}

Through education many patients and parents of affected children have adapted to managing episodes of pain effectively at home using a combination of relaxation and distraction techniques, reassurance, massage etc and often have a supply of analgesia at home. This will usually include paracetamol, a non-steroidal anti-inflammatory and codeine-based analgesic.

Parents are advised to seek medication attention in the event of concerning symptoms, including sepsis, potential life threatening complications e.g., splenic sequestration and if pain response is suboptimal. Attendance on the day unit facility or emergency department is encouraged.

Patients and parents have access to telephone advice in the community or from the hospital. These basic principles of management have 
been summarised previously and form the basis on which many hospital pain management policies have been based.

The principles of hospital management include rapid effective analgesia, which is sustained until pain settles, and then graded withdrawal as the pain improves.

Careful monitoring for the side effects of analgesics by nursing and medical staff is paramount. An awareness of additional sickle cell related complications is important with prompt intervention in the event of a significant complication. Many patients will have complex needs and a multidisciplinary approach to managing these patients is recommended.

There is no single analgesic, which is acceptable and sustainable in all settings. Injected opiates are often used but are uncomfortable and may lead to increasing reliance on unnecessarily high doses of opiates during childhood and adolescence with potential complications in later adult life. Injectable opiates particularly when given intravenously are also often associated with delays in administration particularly in receiving the first dose in the acute setting. Furthermore the need for repeated cannulation is unacceptable for most families.

There are few published data comparing different analgesia regimens in children. Previous studies however have demonstrated that oral controlled release morphine (MST) is an effective alternative to IV morphine. Diamorphine is a potent opiate, which is highly soluble. It is rapidly effective by the intranasal route and has been used for rapid and effective analgesia in children presenting to casualty with trauma. We developed an analgesia regimen using intranasal diamorphine for immediate non-injected analgesia and oral morphine and MST for sustained pain relief.

Figure 1 demonstrates this pathway. This was developed to ensure all children were managed consistently with rapid administration of a short acting opiate that could easily be administered by nursing staff following an initial assessment. An audit of this pathway has demonstrated that the majority of children can be managed with an oral regime of morphine. Short acting morphine (oromorph) needs to be given proactively and more frequently during the first 6-12 hours of the pain event.

We add a long acting morphine (MST) if the pain is not well con-

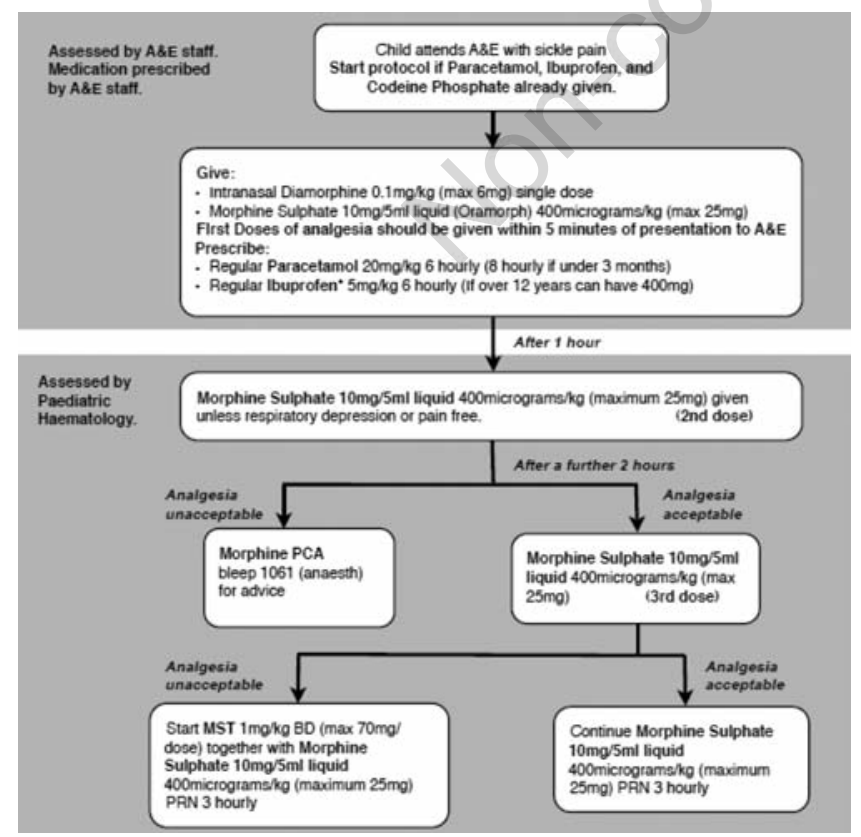

Figure 1. Royal London Hospital paediatric sickle cell pain management protocol.

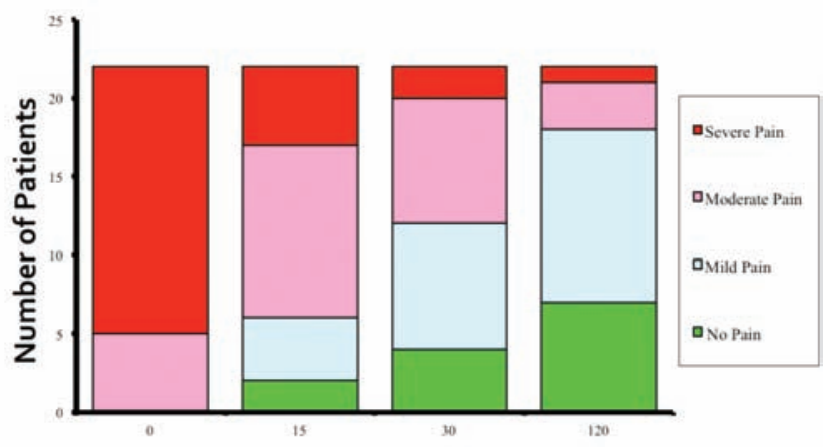

Time from Emergency Department Arrival

Table 1. Observation of pain scores in children treated according to the protocol following arrival in the emergency department.

trolled and this is a very effective analgesic for short-term background pain control (1-7 days). This pathway was demonstrated to rapidly reduce pain scores following implementation in the emergency department, see Table 1.

For management of more complicated sickle cell events associated with pain $e . g$., acute chest syndrome, intravenous administration of morphine or fentanyl given via patient controlled / nurse controlled analgesia (PCA/NCA) is preferred.

The regular observations are an integral part of this pathway with hourly observations recommended for the first 24 hours then two hourly thereafter.

Involvement of specialist teams including the pain team is encouraged and validated age appropriate pain scoring methods employed. Once pain settles and oromorph requirement falls to less than 4 doses in 24 hours then the MST can be reduced. Opiates are discontinued prior to discharge from hospital.

Other medication to manage nausea, constipation, and pruritis should be prescribed; in addition to naloxone, should respiratory depression occur. With careful monitoring the risk of significant side effects related to opiate analgesia can be avoided and there is no risk of opiate dependency if acute painful events are managed according to these principles.

\section{Conclusions}

Sickle cell pain is a complex but frequently experienced symptom. Acute painful events in children can be managed effectively in the community with appropriate support and education.

If hospital management is required, rapid access to a consistent, reliable and safe analgesic pathway is recommended to ensure a good standard of care.

Use of oral opiates in addition to short acting easily administrable forms of analgesia and strict adherence to protocolised monitoring will enable the acute event to be well managed and the negative pain experience minimised.An important part of the outpatient evaluation is determining the impact pain events are having on the child's quality of life.

Addressing psycho-social aspects, defining and modifying precipitating factors, if any are identified, and having a holistic approach to pain management is helpful.

Education and use of self management techniques can also be productive. Use of sickle modifying interventions such as hydroxycarbamide therapy or transfusion therapy can have a significant impact on reducing the severity and frequency of the acute pain event. 


\section{References}

1. Telfer P, Criddle J, Sandell J, Davies F, Morrison I, Challands J. Intranasal diamorphine for acute sickle cell pain. Arch Dis Child 2009;94:979-980.

2. Rees DC, Olujohungbe AD, Parker NE et al. Guidelines for the management of the acute painful crisis in sickle cell disease. British Journal of Haematology 2003;120:744-52.

3. Lucas SB, et al. A sickle cell crisis? A Report of the National Confidential Enquiry into patient Outcome and Death. NCEPOD 2008
4. Franck L, Treadwell M, Jacob E. Assessment of sickle cell pain in children and young adults using the adolescent paediatric pain tool. Journal of pain and symptom management 2002:114-120.

5. Smith W, Bovbjerg V, Penberthy L. Understanding pain and improving management of sickle cell disease: the PISCES Study. Journal of the National Medical association 2005;97:183-193.

6. Ballas Sk, Kramer SA. Acute sickle cell pain episodes: evolution of the clinical picture. J Pain 2001;2:18.

7. Van Beers E, van Tuijn C, Nieuwkerk P. Patient controlled analgesia versus continuous infusion of morphine during vaso-occlusive crisis: a randomised controlled trial. Am J Hematol 2007;82:955960 . 